\title{
Determinants of and barriers to adoption of digital therapeutics for mental health at scale in the NHS
}

\author{
Matt G Williams, ${ }^{1}$ Richard Stott, ${ }^{2}$ Nicki Bromwich, ${ }^{3}$ Susie K Oblak, ${ }^{4}$ \\ Colin A Espie, ${ }^{5}$ James B Rose ${ }^{1}$
}

- Additional material is published online only. To view, please visit the journal online (http://dx.doi.org/10.1136/ bmjinnov-2019-000384).

${ }^{1}$ Clinical Innovation Adoption, Oxford Academic Health Science Network, Oxford, UK

${ }^{2}$ Department of Psychology, Institute of Psychiatry

Psychology and Neuroscience, King's College, London, UK

${ }^{3}$ Strategic and Industry Partnerships, Oxford Academic Health Science Network, Oxford UK

${ }^{4}$ UK Directorate, Big Health, London, UK

${ }^{5}$ Nuffield Department of Clinical Neurosciences, Sleep and Circadian Neuroscience Institute, University of Oxford, Oxford, UK

\section{Correspondence to}

Matt G Williams, Oxford

Academic Health Science Network, Oxford OX4 4GA, UK: Matt.Williams@OxfordAHSN.org

Received 19 August 2019 Revised 14 January 2020 Accepted 18 April 2020 Published Online First 7 May 2020

\begin{abstract}
Objectives Although it is widely acknowledged that digital therapeutics will play a significant role in the future delivery of healthcare, the method by which these innovations may be commissioned by the National Health Service, at scale, remains unclear. This study explores English health commissioners' experiences of, and attitudes towards, adopting digital healthcare innovations.
\end{abstract}

Methods 37 commissioners and decisionmakers from a range of sectors within the health profession were invited to participate in structured interviews to gather relevant examples of experience and opinions. 14 commissioners participated and the notes from the resulting interviews were interpreted using qualitative content analysis. Controlled interpretation was applied to the categorised responses, paraphrasing and classifying into themes. Results Common determinants and barriers to adoption of digital mental health expressed during the interview process included commissioners' lack of familiarity with technology, information governance, digital literacy of users and decision-makers.

Conclusions Recognition of identified barriers may be helpful for innovators when developing engagement and market access strategies and suggest areas for focus and further development within the commissioning process to accommodate digital health solutions.

\section{INTRODUCTION}

Digital therapeutics (DTx) and mobile health (mhealth) products are becoming widely accepted as necessary for the future of efficient healthcare service delivery. ${ }^{1-4}$ Significant advances both in computing power, access to smart devices and connectivity over the last decade have given rise to an entire industry capable of transforming the way healthcare is delivered. These digital health solutions have the potential to expand access to care and enhance our understanding of populations, pathology and how services can be improved for patients and service users at scale. ${ }^{5}$

It is estimated that there are over 327000 different health apps available to download supporting over 240 different health conditions, although it was estimated that $83 \%$ of all health app downloads related to only 43 health apps in the UK. ${ }^{6}$ Only a small proportion of these are likely to be considered DTx which focus on preventing, managing or treating health conditions; either alone or in conjunction with non-digital practices.

The adoption of DTx solutions is complex, often involving multiple stakeholders from different disciplines, numerous decision-making processes and a series of personal or organisational value judgements. ${ }^{8-10}$ Although the National Health Service (NHS) Apps Library offers an array of approved digital products for a range of health issues and conditions, adoption of these apps has been variable across the NHS in England. ${ }^{11}$ This has recently been attributed, in part, to unclear pathways to market and an absence of structured processes around evaluation and commissioning of digital health products as well as financial, regulatory and security hurdles. ${ }^{5} 1213$ Clinician and individuals' concerns relating to data privacy, equality of access, clinical efficacy and safety are also significant potential barriers facing the adoption of DTx solutions. ${ }^{7}$

The recently published Accelerated Access Review acknowledges these challenges, yet also recognises opportunities for more rapidly adopting transformative 
innovations into the NHS to improve patient outcomes. ${ }^{12}$ Serious efforts are now being made to provide clarity and process for both digital innovators, healthcare professionals and patients around adoption of digital health solutions. ${ }^{714}$

The NHS is made up of hundreds of separate but linked organisations, and the burden of managing complex interactions and data flows between trusts, systems and individuals too often falls on patients. Currently, much NHS technology relies on systems designed for a pre-internet age, and data does not follow a patient around the system. Change has been slow because responsibility for digital, data and tech has been split across multiple agencies, teams and organisations. It is possible that a new organisation, NHSX, will address this by bringing together experts in technology, digital, data and cyber security to develop world-class digital services, to build on the NHS Long Term Plan. ${ }^{4} 15$

Mental health is one area where DTx solutions are being developed and trialled to expand access to treatments and potentially reduce the need for face-to-face therapies. Using a well-evidenced, digital cognitivebehavioural therapy (CBT) based tool for treating insomnia (www.sleepio.com) as a technology exemplar, the work described herein sought to evaluate the challenges and barriers to identifying, commissioning and implementing novel digital solutions for mental health at scale across a population.

\section{METHODS}

The Oxford Academic Health Science Network and Big Health (the developers of the DTx exemplar, Sleepio) were awarded a grant by Innovate UK to explore how a DTx product might be offered at population scale over a period of twelve months. One of the expected outcomes from the project would be the creation of an effective blueprint for commissioners to adopt and roll out future DTx products. As part of one of the work packages within this project, 37 commissioners and senior decision-makers likely to be involved in commissioning digital mental health solutions across the South East of England were invited by email to participate in this study. Fourteen stakeholders (38\%) responded and took part in the interview programme.

Eight (57\%) respondents were interviewed face to face and in $6(43 \%)$ cases, when this was not possible, a teleconference call was conducted. All interviews took place in the workplace.

All interviews were semistructured and presented consistent questions to all participants. The interview guide has been included as online supplementary material. Each interview lasted between 45 and $75 \mathrm{~min}$. Each interview covered questions on:

- The need for innovation in digital health.

- Respondents' experience of commissioning digital health products and DTx.
- Respondents' perceptions of types of arguments to support adoption.

- Local/regional decision-making processes.

- Respondents' experience of implementing digital health programmes at scale.

In addition, participants were given some multiple choice and ranking exercises to gauge their perceptions of different commissioning models for the case study DTx (www.sleepio.com). Rankings were anchored with case examples of maximum and minimum ratings. For each interview, there were three researchers present, including the corresponding author, to manage questioning and recording of responses. Although they would provide a permanent and accurate record of the interviews, audio recordings were considered potentially off putting for participants and contemporaneous notes were taken by one member of the panel during each interview with the respondent's consent. Both genders were represented on each interviewing panel.

Interview notes were transcribed and anonymised after completion and were interpreted using the method of qualitative content analysis as described by Mayring. ${ }^{16}$ Qualitative content analysis is popular in many areas of psychological and sociological research offering a systematic and sequential model for analysis also finding a place in case study research. Its rule-based nature makes the process easy to learn and easily translatable to a variety of research questions. It was deemed to be the most appropriate model for this research question, which was relatively well defined, and where more open procedures, for example, grounded theory may not have been suitable.

In brief, the process started with decontextualisation of the responses using an inductive coding process. Iterative cleansing of the coded ideas then afforded an array of concepts which were then grouped and categorised by the research team collectively, first to reported phases of the digital innovation adoption process and then into homogenous themes and subthemes. A triangulation phase was performed with the research team to ensure that thematic categorisation was justified and consistent between respondents. The final models bring together all themes and related subthemes into a single framework relevant to a specific part of the innovation adoption process. This process provided a systematic approach to allow valid inferences to be drawn from the data that relate to practical challenges and implications around the adoption of DTx.

\section{RESULTS}

\section{Participant characteristics}

Respondents' backgrounds ranged from general practitioner (GP) Mental Health Leads, clinical commissioning groups (CCGs) Senior Commissioning Managers, Heads of Medicines Optimisation, Planned Care Leads and Assistant Directors of Public Health. Respondents' knowledge and background are characterised best by four professional roles (see figure $1 \mathrm{~A}$ ). 
A

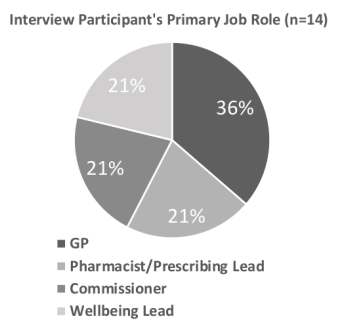

B

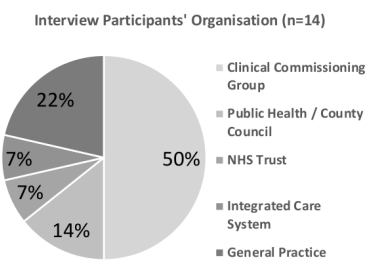

c

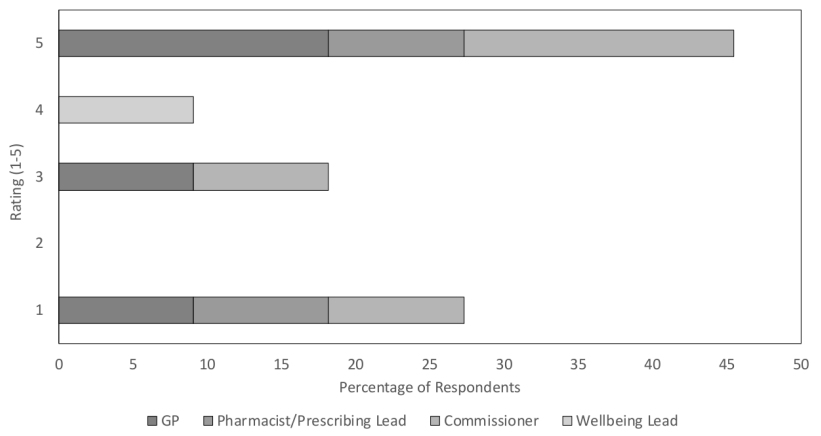

Figure 1 Backgrounds and primary job role of the sample engaged and respondents' rating of their level of experience of commissioning digital health products. Graphs showing breakdown by percentage of complete sample $(n=14)$. GP, general practitioner; NHS, National Health Service.

Half of the respondent sample were affiliated with CCGs (see figure 1B).

Range of experience among interviewees in commissioning digital health innovations varied widely. Just under half of the respondents rated their experience of commissioning digital health products as the highest ranking (5; see figure $1 \mathrm{C}$ ). Those who rated themselves as having less experience often explicitly stated that this was due to a lack of opportunity, rather than enthusiasm for engagement with such technology. Examples such as Kooth, Healios and Big White Wall were cited as recently commissioned or trialled products. ${ }^{17-19}$ Some digital health products described had been designed, commissioned and rolled-out locally. Self-development

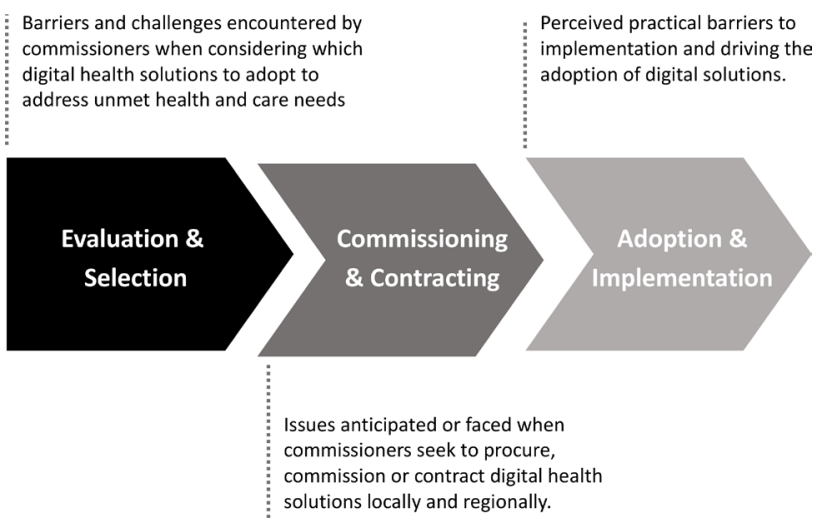

Figure 2 The three key phases of adoption of digital health solutions drawn from coding and categorisation of responses.

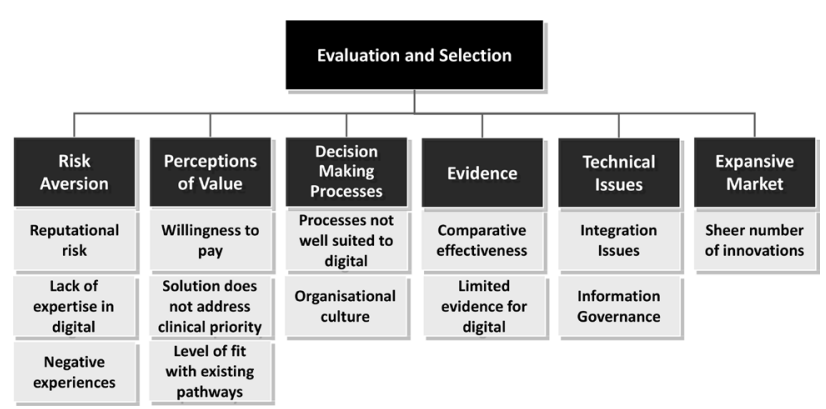

Figure 3 Key themes derived from the process of qualitative content analysis of interview responses related to evaluating and selecting digital health innovations.

approaches were considered appealing as the organisation retained "control around all aspects of the bespoke project and [we] were able to engage residents in design". (Commissioner, Public Health)

\section{Phases of commissioning digital health solutions}

Several themes emerged from the information collected from respondents. A number of options for grouping and ordering the thematic issues were considered. A chronological approach was chosen as the issues expressed could, with some exceptions, be placed within the current commissioning journey of a new health innovation; from initial evaluation and selection, to the process of commissioning and creating contracts, to the final phase of rollout and implementation (see figure 2).

Phase one: evaluation and selection

The key themes derived from the process of qualitative content analysis of interview responses related to evaluating and selecting are summarised in figure 3 .

Digital innovation was commonly described as being risky. Not only in terms of uncertainty around potential outcomes and take-up, but for commissioners personally. Tightly controlled budgets meant that redirection of funding from an existing programme was open to greater scrutiny and several participants gave examples when they, or their department, had been criticised for funding projects that had not delivered.

Four (29\%) commissioners expressed support and interest in DTx innovations; however, they suggested that the funding should be seen as the responsibility of other organisations/agencies/departments, for example, "shouldn't this sit under 'prevention'?" (Commissioner, Clinical Commissioning Group)

Three (21\%) commissioners stated that they would, personally, be supportive of adopting innovations but believed they would not receive the support of decision-making colleagues particularly in organisations with a culture of slow adoption.

Concerns were expressed around the role of the commissioner, as a non-technical expert, in deciding which digital health products-from the myriad available-to promote and recommend locally. Three 


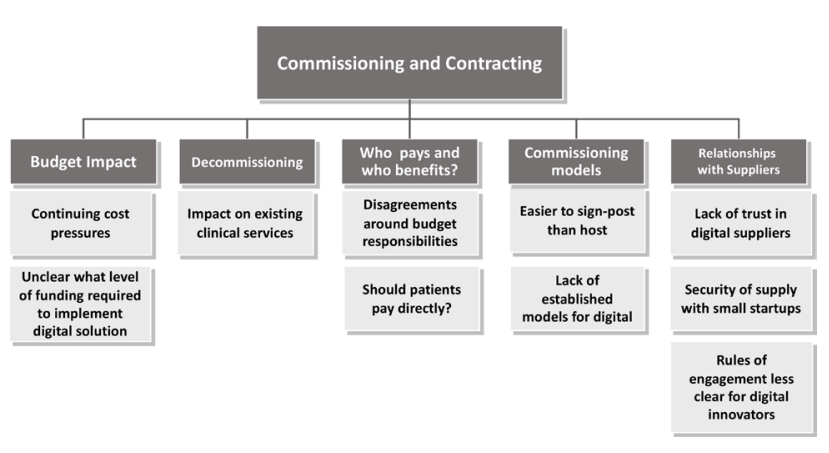

Figure 4 Key themes derived from the process of qualitative content analysis of interview responses related to commissioning and contracting digital health innovations.

(21\%) participants expressed support for the creation of a central, decision-making body to evaluate such products, preferably producing a pre-evaluated and approved 'menu' of DTx innovations.

The evidence for clinical effectiveness of Sleepio, the technology exemplar being used, was particularly strong ${ }^{20-29}$ and as such was seen positively by the respondents. Evidence was considered a particularly important determinant of selection and downstream adoption, particularly compared against evidence for non-digital incumbent treatments.

It was perceived by three (21\%) respondents that digital health solutions also carry an additional burden of risk in terms of "patient data security" (GP, General Practice), "technical integration issues and information

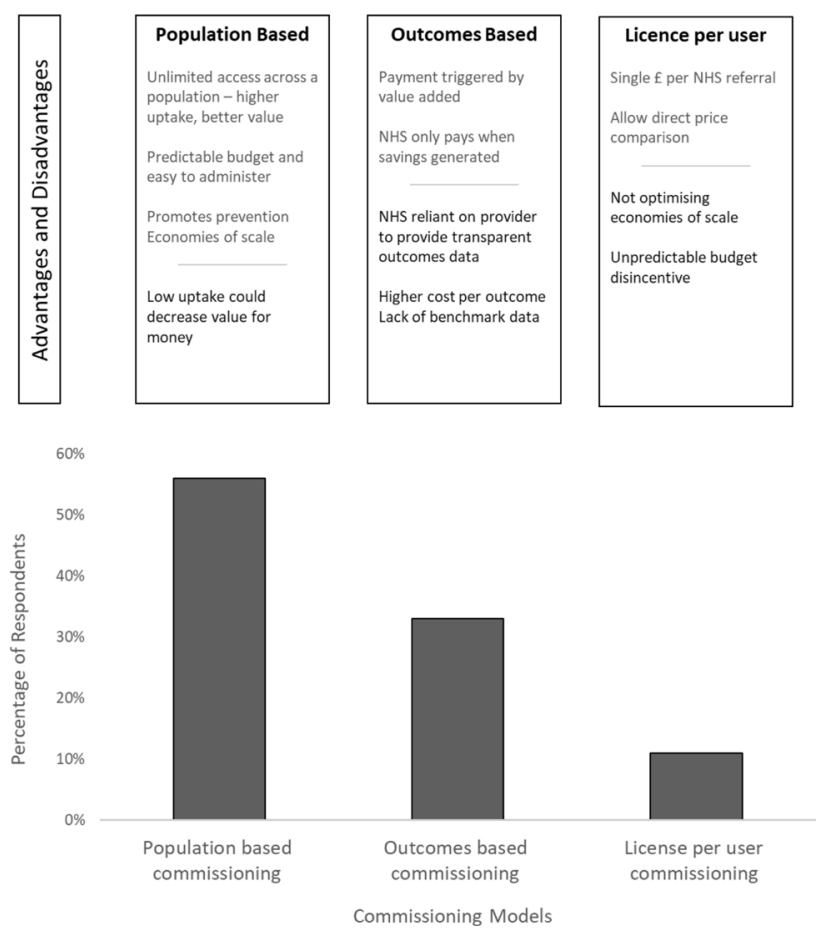

Figure 5 Respondent preferences to three commissioning models presented for the case study digital health solution. Figure shows percentages of respondents favouring each model and the advantages and disadvantage raised during the interview. NHS, National Health Service. governance". (Commissioner, Clinical Commissioning Group)

Phase two: commissioning and contracting

The key themes derived from the process of qualitative content analysis of interview responses related to commissioning and contracting are shown in figure 4.

Three commissioning models were suggested to participants (figure 5). The most commonly supported commissioning model, from those offered, was population-based commissioning; a single fee to allow population-wide uptake. Five (36\%) participants selected this as the model most likely be adopted in practice; however, participants noted that budget impact and related factors were also important considerations.

Outcomes-based commissioning was selected as the preferred model by three (21\%) commissioners, including two GPs. However, it was noted that this model was highly reliant on access to robust data and measurement systems. All three commissioners raised concerns around data quality and collection.

A variation of the outcomes-based commissioning model, proposed by two (14\%) participants, was a 'risk-share' approach, based on a rebate in situations where agreed outcomes were not met.

The principle of license-per-user commissioning was not well supported and selected by only one (7\%) participant. The reason for this being unfavourable to many was that it would be unlikely to provide economic predictability when considering rollout across a wide geographical area.

A more acceptable approach to commissioning DTx products, suggested by four commissioners $(28 \%)$, would be to integrate them into existing local programmes of support for residents offering a range of well-being services, or to signpost potential users to a DTx product, minimising risk and cost.

Although cost saving was a primary aspiration of commissioners, decisions to fund were also largely based on the comparative quality of care, or outcomes offered, relative to the incumbent product or service. Several commissioners suggested that compelling qualitative data from patients expressing positive sentiments and a demand for a product would be taken seriously and strengthen any business case submitted.

One respondent described that if committing to a digital health implementation programme "inadequate funding could have significant implications on its viability. Conversely, overly generous funding could lead to raised expectations and accusations of profligacy". (Commissioner, Public Health)

Phase three: adoption and implementation

The key themes derived from the process of qualitative content analysis of interview responses related to adoption and implementation are presented in figure 6.

A commonly expressed view was that digital health products would be acceptable to younger, but not older patients. Such assertions were based on the experiences, 


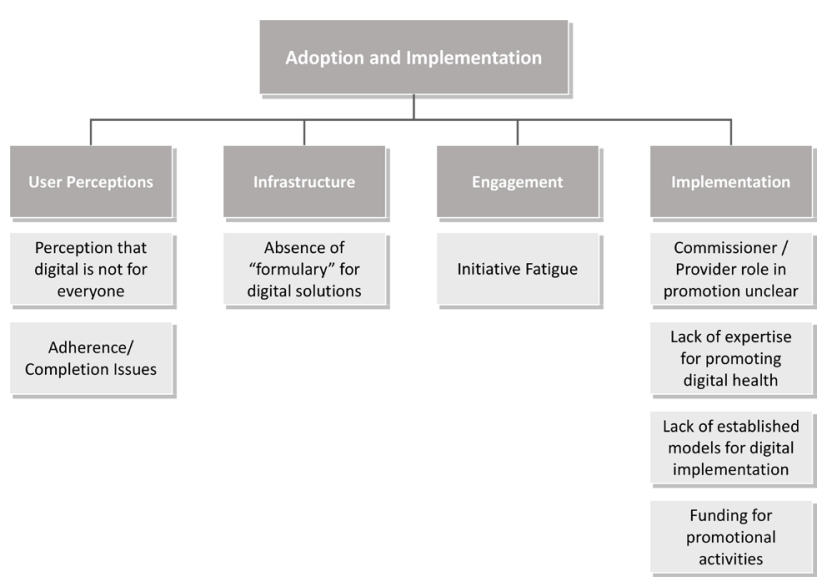

Figure 6 Key themes derived from the process of qualitative content analysis of interview responses related to adoption and implementation.

and in three (21\%) instances on the preconceptions, of the individual commissioners involved. Concerns about those with learning or language difficulties or lack of access to technology were also proposed as potential barriers to both implementation and commissioning.

Unlike the existing situation with pharmaceuticals, no examples of established forums or working groups for digital health implementation were given by the respondents. Comparisons were drawn with prescribing groups, drug and therapeutic committees for medicines optimisation which currently exist.

Potential issues around adherence were expressed by two (14\%) respondents. Unlike medicines, there are "...no obvious moments, such as the ordering of repeat prescriptions" (Pharmacist, Clinical Commissioning Group), to prompt reviews or indicate to the clinician that a course of treatment had been completed. Although this placed responsibility in the hands of patients, which may be considered empowering, concerns were expressed that such products might be used inconsistently, or not at all.

The respondents with main role as GP reported that the sheer number of new initiatives that clinicians, managers and commissioners are expected to engage with is deemed to be too high and commonly expressed as 'initiative fatigue'. This concern was not specific to digital adoption.

The practical question as to how digital health products could be disseminated to a wider audience was expressed as part of these conversations. Without advertising budgets or the involvement of company representatives, commissioners were unable to describe alternative ways to promote such products and suggested this was beyond the scope of their role.

\section{DISCUSSION}

This work outlines how difficult it is for healthcare leaders and commissioners to initiate, execute and sustain changes in the NHS. This work has looked to explore some of the most significant barriers to the various stages of adopting DTx technologies for mental health at scale. It highlights 'digital-specific' issues which increase the complexity around selection, commissioning and implementation.

Commissioners' lack of familiarity with technology was commonly expressed. This might be resolved by the development of a central, decision-making body to evaluate and approve new digital health and DTx products. The research team acknowledge the existence of three potential resources fitting this description, for example, ORCHA, NHS Apps Library, and Our Mobile Health. ${ }^{711} 30$

Formularies and other local structures to allow clinicians to discuss and receive updates about pharmaceutical medications and other treatments have developed over time. These existing structures might, in time, allow DTx to receive similar attention alongside existing treatments. However, the development of parallel structures focussing on DTx could allow those with technical experience and understanding to aid clinicians to safely navigate and build their knowledge in this emerging area.

Commonly held attitudes and perspectives around DTx among commissioners require challenge, particularly those around the age profile of potential users. The misconception that young people represent the key audience, preferring or demanding digital solutions, is widespread despite evidence to the contrary. ${ }^{2028} 31$

Furthermore, developers of digital innovations were described, by several commissioners, as being unaware of the acceptable levels of evidence required to make a sufficiently robust case when approaching commissioners, though the authors acknowledge that clinical evidence is being addressed through the NICE Evidence for Effectiveness Framework. ${ }^{14}$ Over time it may also be necessary for specific forums for digital health, potentially requiring specialised knowledge, to be established locally or regionally.

In addition, this study reported interesting observations in terms of preferences around commissioning models. Few commissioners have a wealth of experience of commissioning digital health solutions or the models by which this is best done. While outcomesbased commissioning was popular, a significant hurdle was seen by many in terms of the associated increases in data collection, analysis and reporting. However, as connectivity and data integration in healthcare advances, such burdens in capturing outcomes are likely to be reduced which may change the landscape and commissioners' preferences around commissioning models.

Population-based commissioning was deemed the most acceptable of the models suggested, though the potential for poor take-up and reliance on effective dissemination made this option potentially risky for commissioners whose role rarely requires expertise in marketing or population engagement.

While digital health and mhealth are broad technology areas, comprising thousands of solutions 
offering different degrees of transformative change, it is proposed that the barriers faced are likely to be common and consistent, particularly in the areas related to the processes around decision-making in commissioning organisations, and that the learning presented could be applied to a range of other digital health products.

It is important to note the limitations in this study. The sample size, while covering a broad complement of likely stakeholders involved in digital commissioning, was small. In addition, the case study DTx product, a digital CBT solution for insomnia (www.sleepio.com), was unusual in terms of its strong evidence base, having arrived at the market with eight randomised controlled trials and a host of other peer-reviewed studies. ${ }^{20-29}$ Digital health products are typically launched with limited published data around safety and effectiveness and, as such, it remains difficult to compare potential DTx solutions with incumbent technologies already commissioned.

\section{CONCLUSIONS}

There are few studies that have looked at the specific local and regional issues associated with commissioning digital solutions for well recognised population health needs. This study highlights a number of important barriers likely to be faced by innovators and suppliers of DTx mental health solutions attempting to gain spread and adoption at scale in the NHS. Recognition of such barriers may be helpful for innovators when developing engagement and market access strategies. Further work is most certainly required in understanding this complex and evolving area of commissioning and in supporting commissioners to select, evaluate and implement digital solutions likely to positively transform healthcare ecosystems and improve outcomes for patients.

\section{Twitter James B Rose @OxfordAHSN}

Acknowledgements The authors would like to acknowledge and thank all respondents for their time in supporting this work.

Contributors MGW, NB, SKO and JBR designed the study and prepared the interview questions. MGW, NB and SKO conducted and reported the interviews. MGW, JBR and NB analysed the data and wrote the manuscript with support from RS and CAE.

Funding Funding for this work was provided by Innovate UK through the Digital Health Technology Catalyst fund.

Competing interests However, at the time of publishing, two authors currently hold positions at Big Health, the developer of the Sleepio product.

Patient consent for publication Not required.

Provenance and peer review Not commissioned; externally peer reviewed.

Data availability statement Data are available upon reasonable request.

\section{REFERENCES}

1 Barton CL. Mobile health technologies and global markets 2014.

2 Deloitte. Digital health in the UK 2015.

3 European Commission. Enabling the digital transformation of health and care in the digital single market 2018.
4 NHSX. NHSX Homepage.

5 Steinhubl SR, Muse ED, Topol EJ. The emerging field of mobile health. Sci Transl Med 2015;7:283rv3.

6 Aitken M, Clancy B, Nass D. The growing value of digital health: evidence and impact on human health and the healthcare system. Inst Rep IQVIA Inst Hum Data Sci 2017:1-76.

7 ORCHA. Orcha - The Challenge - Getting the Right Apps to the Right People at the Right Time, 2019. Available: https:// www.orcha.co.uk/the-challenge/

8 Greenhalgh T, Robert G, Macfarlane F, et al. Diffusion of innovations in service organizations: systematic review and recommendations. Milbank Q 2004;82:581-629.

9 Greenhalgh T, Wherton J, Papoutsi C, et al. Beyond adoption: a new framework for theorizing and evaluating Nonadoption, abandonment, and challenges to the scale-up, spread, and sustainability of health and care technologies. J Med Internet Res 2017;19:e367.

10 Accelorator NI. NHS Innovation Accelerator : Understanding how and why the NHS adopts innovation. NHS Innov 2018.

11 NHS. Nhs APP library, 2019. Available: https://www.nhs.uk/ apps-library/

12 Independent. Accelerated Access Review - Final Report 2016.

13 He D, Naveed M, Gunter CA, et al. Security concerns in android mHealth Apps. AMIA Annu Symp Proc 2014;2014:645-54.

14 NICE. National Institute for health and care excellence evidence standards framework for digital 2019;35.

15 NHS. The NHS long term plan_ digitally enabled care. NHS Long Term Plan, 2019.

16 Mayring P. Qualitative Content Analysis. Forum Qual Sozialforsch / Forum Qual Soc Res Vol 1, No 2 Qual Methods Var Discip I Psychol - 1017169/fqs-121089. Available: http:// www.qualitative-research.net/index.php/fqs/article/view/1089/ 2385 [Accessed 30 Jun 2000].

17 Big white wall. big white wall, 2019. Available: www. bigwhitewall.com

18 Kooth. Kooth, 2019. Available: www.kooth.com

19 Healios. Healios, 2019. Available: www.Healios.org.uk

20 Espie CA, Emsley R, Kyle SD, et al. Effect of digital cognitive behavioral therapy for insomnia on health, psychological wellbeing, and sleep-related quality of life: a randomized clinical trial. JAMA Psychiatry 2019;76:21-30.

21 Freeman D, Sheaves B, Goodwin GM, et al. Effects of cognitive behavioural therapy for insomnia on the mental health of university students: study protocol for a randomized controlled trial. Trials 2015;16:236.

22 Freeman D, Sheaves B, Goodwin GM, et al. The effects of improving sleep on mental health (OASIS): a randomised controlled trial with mediation analysis. Lancet Psychiatry 2017;4:749-58.

23 Luik AI, Bostock S, Chisnall L, et al. Treating depression and anxiety with digital cognitive behavioural therapy for insomnia: a real world NHS evaluation using standardized outcome measures. Behav Cogn Psychother 2017;45:91-6.

24 McGrath ER, Espie CA, Power A, et al. Sleep to lower elevated blood pressure: a randomized controlled trial (slept). Am J Hypertens 2017;30:319-27.

25 Bostock S, Luik AI, Espie CA. Sleep and productivity benefits of digital cognitive behavioral therapy for insomnia: a randomized controlled trial conducted in the workplace environment. J Occup Environ Med 2016;58:683-9.

26 Pillai V, Anderson JR, Cheng P, et al. The anxiolytic effects of cognitive behavior therapy for insomnia: preliminary 
results from a Web-delivered protocol. J Sleep Med Disord 2015;2:1017.

27 Cheng P, Luik AI, Fellman-Couture C, et al. Efficacy of digital CBT for insomnia to reduce depression across demographic groups: a randomized trial. Psychol Med 2019;49:491-500

28 Espie CA, Kyle SD, Williams C, et al. A randomized, placebocontrolled trial of online cognitive behavioral therapy for chronic insomnia disorder delivered via an automated mediarich web application. Sleep 2012;35:769-81.
29 Espie CA, Kyle SD, Miller CB, et al. Attribution, cognition and psychopathology in persistent insomnia disorder: outcome and mediation analysis from a randomized placebocontrolled trial of online cognitive behavioural therapy. Sleep Med 2014;15:913-7.

30 Our Mobile Health. Our mobile health, 2019. Available: www. ourmobilehhealth.com

31 Bol N, Helberger N, Weert JCM. Differences in mobile health APP use: a source of new digital inequalities? The Information Society 2018;34:183-93. 